\title{
Integral Action Controllers for Systems with Time Delays $^{\star}$
}

\author{
Hitay Özbay ${ }^{1}$ and A. Nazli Gündeş ${ }^{2}$ \\ 1 Dept. Electrical \& Electronics Eng., Bilkent Univ., Ankara, 06800 Turkey, \\ hitay@bilkent.edu.tr \\ 2 Dept. Electrical \& Computer Eng., Univ. of California, Davis, CA 95616, U.S.A., \\ angundes@ucdavis. edu
}

Summary. Consider a stabilizing controller $C_{1}$ for a given plant $P$. If $C_{1}$ and $P$ do not have any zeros at the origin, then one can use a cascade connected PI (proportional plus integral) controller $C_{p i}$ with $C_{1}$ and keep the feedback system stable. In this work we examine the allowable range of the integral action gain in $C_{p i}$, and discuss how $C_{1}$ should be chosen to maximize this range for systems with time delays.

\section{Introduction}

In the design of feedback controllers it is often desirable to use an integrator to be able to track constant reference signals. For example, internal model principle says that the controller must include a copy of the reference signal (or disturbance) generator in order to have a robust tracking (or disturbance rejection), see e.g. [2, 4, 7]. Typically, the reference generator $G_{r}(s)$ is an unstable system: an integrator (resp. oscillator) if the reference is a constant (resp. a sinusoidal signal). One way to achieve robust asymptotic tracking (or disturbance rejection) is to append $G_{r}$ to the plant $P$ and then design a controller $C_{o}$ for the combined "plant" $G_{r} P$. Thus $C=C_{o} G_{r}$ is a stabilizing controller for $P$ and it achieves the performance objectives, see e.g. [1] 16] for more details.

In this chapter we consider the dual problem: first design a stabilizing controller for the plant, then append a PI term to this controller. A similar problem has been discussed in [3] for finite dimensional systems. Briefly, the problem we deal with can be stated as follows: let $C_{1}$ be a stabilizing controller for a time delay system $P$, and append (in the form of a cascade connection) $C_{p i}(s)=\frac{\left(s+k_{i}\right)}{s}$ to $C_{1}$. Note that the proportional gain of the PI controller is set to unity; this is without loss of generality since a non-unity gain can be absorbed into $C_{1}$. Assume that $P$ and $C_{1}$ do not have any zeros at the origin. Then, there exists $k_{i}$ such that the feedback system is stable. We examine the range of allowable $k_{i}$, and discuss the problem of designing an optimal $C_{1}$ so that this range is maximized.

We should indicate that rather than the cascade PI-controller connection to be discussed here, a two-stage parallel connection of controllers is also possible. For example, as before, let $C_{1}$ be a a stabilizing feedback controller for a given plant $P$. If the PI part of the controller,

\footnotetext{
* This work was supported in part by TÜBITAK (grant no. EEEAG-105E156).
} 
$C_{p i}$, is a stabilizing controller for the new plant $P\left(I+C_{1} P\right)^{-1}$, then the parallel connection of the controller, $C_{p i}+C_{1}$, is a stabilizing controller for the original plant $P$, see 99 , 14 , 16]. One can study the problem choosing the best $C_{1}$ so that the allowable range of $k_{i}$ is maximum. We leave this problem aside, because the techniques to be used in such a study would be similar to the approach taken in this chapter for the cascade connection of the controllers.

This chapter is organized as follows: stability of the feedback system under cascade connection of the PI controller is investigated in Section 2 Design of $C_{1}$, maximizing the allowable range of the integral gain, is discussed in Section 3 Concluding remarks are made in Section 4

Notation used here is standard. In particular, the norm sign $\|\cdot\|$ stands for the $\mathcal{H}_{\infty}$ norm $\|\cdot\|_{\infty}$ whenever the argument is in $\mathcal{H}_{\infty}$.

\section{Feedback System Stability Under Cascade Connection of the PI Controller}

Consider the feedback system shown in Figure1 with an $r$ input $r$ output plant whose $r \times r$ transfer matrix is $P(s)$. The $r \times r$ controller transfer matrix is $C(s)$. Assume that $P$ is full rank. The feedback system is said to be stable if $C(1+P C)^{-1}, P C(1+C P)^{-1}, C(I+$ $P C)^{-1} P, P(1+C P)^{-1}$ are in $\mathcal{H}_{\infty}^{r \times r}$. In this case, we say $C \in \mathcal{S}(P)$, where $\mathcal{S}(P)$ is the set of all controllers stabilizing the feedback system with plant $P$.

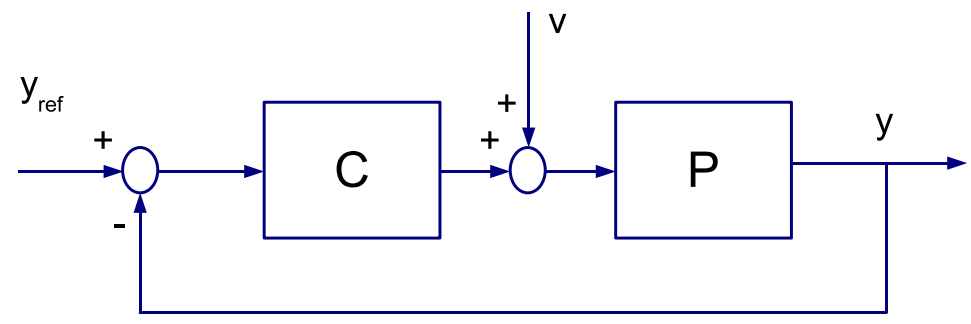

Fig. 1. Feedback System

Let $C_{1}$ be in $\mathcal{S}(P)$ and consider the cascade connection $C=C_{1} C_{x}$ for some $C_{x}$. The result stated below as Theorem 1 addresses the following question: Is the closed-loop system still stable if $C=C_{1} C_{x}$, i.e. do we have $C \in \mathcal{S}(P)$ ?

Theorem 1. Let $P$ be a given $r \times r$ plant and let $C_{1} \in \mathcal{S}(P)$. Assume that $P$ and $C_{1}$ are full rank and define the complementary sensitivity function for the feedback system with $C=C_{1}$ as $T_{1}:=P C_{1}\left(I+P C_{1}\right)^{-1}$. Then, we have the following two results:

a) If $C_{y}:=C_{x}-I$ stabilizes $T_{1} \in \mathcal{H}_{\infty}^{r \times r}$, then

$$
C=C_{1} C_{x} \in \mathcal{S}(P) \text {. }
$$

b) Let $P$ and $C_{1}$ have no transmission-zeros at the origin. Choose any $\hat{K}_{P}, \hat{K}_{D} \in \mathbb{R}^{r \times r}$, and $\tau \in \mathbb{R}_{+}$. Define $\Psi \in \mathcal{H}_{\infty}^{r \times r}$ as 


$$
\Psi(s)=\frac{T_{1}(s) T_{1}(0)^{-1}-I}{s}+T_{1}(s)\left(\hat{K}_{P}+\frac{\hat{K}_{D} s}{\tau s+1}\right) .
$$

Then for $\rho \in \mathbb{R}_{+}$satisfying

$$
\rho<\|\Psi\|^{-1}=: \psi^{-1}
$$

the controller $C=C_{1} C_{\text {pid }} \in \mathcal{S}(P)$, where $C_{\text {pid }}$ is a PID-controller given by $C_{\text {pid }}=$ $I+\hat{C}_{\text {pid }}$, where

$$
\hat{C}_{p i d}=\rho\left(\hat{K}_{P}+\frac{T_{1}(0)^{-1}}{s}+\frac{\hat{K}_{D} s}{\tau s+1}\right) .
$$

For $\hat{K}_{D}=0$, (4) becomes a PI-controller.

Proof of Theorem 1 is given in the Appendix. By part (a) of this theorem the stabilizing controller $C=C_{1} C_{x}$ gives rise to the following complementary sensitivity $T_{x}=P C(I+$ $P C)^{-1}$ :

$$
\begin{aligned}
T_{x} & =P C_{1}\left(I+C_{x} P C_{1}\right)^{-1} C_{x}=P C_{1}\left(I+P C_{1}+C_{y} P C_{1}\right)^{-1} C_{x} \\
& =T_{1}\left(I+C_{y} T_{1}\right)^{-1}\left(I+C_{y}\right)=\left(I+T_{1}\left(I-C_{x}\right)\right)^{-1} T_{1} C_{x} .
\end{aligned}
$$

If $C_{x}=C_{p i d}$ as in (4) of Theorem 1 (b), then $T_{x}$ in 5 becomes

$$
T=\left(I+T_{1} \hat{C}_{p i d}\right)^{-1} T_{1} C_{p i d},
$$

which can be expressed as

$$
\begin{aligned}
T & =\left(\frac{s}{s+\rho} I+T_{1}(s) \hat{C}_{p i d}(s) \frac{s}{s+\rho}\right)^{-1} T_{1}(s)\left(\frac{s}{s+\rho} I+\frac{s}{s+\rho} \hat{C}_{p i d}\right) \\
& =\left(I+\frac{\rho s}{s+\rho} \Psi(s)\right)^{-1} T_{1}(s) \frac{\rho s}{s+\rho}\left(I+\rho s\left(\hat{K}_{P}+\frac{T_{1}(0)^{-1}}{s}+\frac{\hat{K}_{D} s}{\tau s+1}\right)\right) .
\end{aligned}
$$

Therefore, $T(0)=I$ and

$$
\|T\| \leq\left\|\left(I+\frac{\rho s}{s+\rho} \Psi\right)^{-1}\right\| \cdot\left\|T_{1}(s) \frac{s}{s+\rho}\left(I+\hat{C}_{p i d}\right)\right\| .
$$

Writing $\left(I+\frac{\rho s}{s+\rho} \Psi\right)^{-1}=I-\left(I+\frac{\rho s}{s+\rho} \Psi\right)^{-1} \frac{\rho s}{s+\rho} \Psi$, we obtain

$$
\left\|\left(I+\frac{\rho s}{s+\rho} \Psi(s)\right)^{-1}\right\| \leq 1+\rho \psi\left\|\left(I+\frac{\rho s}{s+\rho} \Psi\right)^{-1}\right\|
$$

and hence, $\left\|\left(I+\frac{\rho s}{s+\rho} \Psi(s)\right)^{-1}\right\| \leq(1-\rho \psi)^{-1}$, and

$$
\|T\| \leq \frac{1}{1-\rho \psi}\left\|T_{1}(s) \frac{s}{s+\rho}\left(I+\hat{C}_{p i d}(s)\right)\right\| .
$$

Now suppose that in the PID-controller $\hat{C}_{\text {pid }}$ we choose $\hat{K}_{P}=0, \hat{K}_{D}=0$. Then by 44, the PI-controller is

$$
C_{p i}(s)=I+\frac{\rho T_{1}(0)^{-1}}{s},
$$

where $\rho \in \mathbb{R}_{+}$satisfies [3, i.e.,

$$
\rho<\left\|\frac{T_{1}(s) T_{1}(0)^{-1}-I}{s}\right\|^{-1}=: \psi_{o}^{-1} .
$$


In this case, the upper-bound on $\|T\|$ given in 80 becomes

$$
\|T\| \leq \frac{1}{1-\rho \psi_{o}}\left\|T_{1} T_{1}(0)^{-1}\right\| \cdot\left\|\frac{s T_{1}(0)+\rho I}{s+\rho}\right\| .
$$

In particular, if $T_{1}(0)=I$, i.e. $C_{1}$ and/or $P$ contain a pole at $s=0$, then

$$
\|T\| \leq \frac{1}{1-\rho \psi_{o}}\left\|T_{1}\right\|
$$

$>$ From the above discussion we see that if $\rho \psi_{o} \ll 1$ then the upper bound of $\|T\|$ is close to $\left\|T_{1}\right\|$.

\section{Design of $C_{1}$ Maximizing the Integral Action Gain}

In this section we discuss the design of $C_{1}$ for the largest allowable range of $\rho$, 9), for a class of single input single output (SISO) plants with time delays. In this case, from 9 we see that $C_{1}$ should be designed to minimize

$$
\psi=\left\|\frac{T_{1}(s) T_{1}^{-1}(0)-1}{s}\right\|_{\infty},
$$

where $T_{1}=P C_{1}\left(1+P C_{1}\right)^{-1}$ and $C_{1} \in \mathcal{S}(P)$.

Solution of this problem will be obtained below in two steps: (i) first we solve the problem for stable plants, then (ii) we extend this solution to cover unstable plants case. In both steps we begin with inner-outer and coprime factorizations of given $P$, then we solve an $\mathcal{H}_{\infty}$ optimization problem. Inner-outer factorizations require finding $\mathbb{C}_{+}$roots of a quasi-polynomial, for which several algorithms exist by now, see e.g. [5] 12, 17] and their references. Using these algorithms and the methods developed for the $\mathcal{H}_{\infty}$ control of general infinite dimensional systems, (see e.g. [6] and [8]) we can solve the problem in step (i) for a large class of time delay systems. We will see that the extension (ii) to unstable plants, with finitely many poles in $\mathbb{C}_{+}$, involves a parameterization of all suboptimal solutions of the problem in (i), and the use of Nevanlinna-Pick interpolation. The mathematical tools for these problems can be found in [6, 11, 15, 18].

\subsection{Stable plants}

In this section we consider stable SISO plants whose inner-outer factorizations are in the form $P=P_{i} P_{o}$ where $P_{i}$ is inner (all-pass) with $P_{i}(0)=1$, and $P_{o}$ is outer (minimum-phase).

Example. Consider the plant with input/output delay, $h>0$, and internal delays

$$
P(s)=\left(\frac{e^{-h s}}{s+2}\right) \frac{(s+1)+2(s-1) e^{-2 s}}{(s+3)+e^{-3 s}} .
$$

Then, the following is an inner-outer factorization:

$$
\begin{aligned}
& P_{i}(s)=-e^{-h s} \frac{(s+1)+2(s-1) e^{-2 s}}{2(s+1)+(s-1) e^{-2 s}} \\
& P_{o}(s)=\frac{-1}{s+2} \frac{2(s+1)+(s-1) e^{-2 s}}{(s+3)+e^{-3 s}}
\end{aligned}
$$


Clearly, $P_{i}(0)=1$, the poles and zeros of $P_{i}$ are symmetric around the Im-axis, and $P_{o}$ contains no poles or zeros in the right half plane.

The set of all stabilizing controllers is parameterized as

$$
\mathcal{S}(P)=\left\{Q /(1-P Q): Q \in \mathcal{H}_{\infty} \text { and } P Q \neq 1\right\} .
$$

Therefore, $C_{1}$ must be in the form $C_{1}=Q_{1} /\left(1-P Q_{1}\right)$, where $Q_{1} \in \mathcal{H}_{\infty}$ is free. Let

$$
Q_{1}(s)=P_{o}^{-1}(s) \frac{Q_{i}(s)}{(1+\varepsilon s)^{\ell}}
$$

where $Q_{i} \in \mathcal{H}_{\infty}$ is the free parameter, $\varepsilon>0$, and $\ell$ is the relative degree of $P_{o}$. Then we have

$$
T_{1}(s)=P(s) Q_{1}(s)=P_{i}(s) \frac{Q_{i}(s)}{(1+\varepsilon s)^{\ell}} .
$$

Hence the problem of maximizing the allowable range of $\rho$ reduces to finding

$$
\begin{aligned}
\psi_{o} & =\inf _{Q_{i} \in \mathcal{H}_{\infty}}\left\|\left(\frac{P_{i}(s)}{(1+\varepsilon s)^{\ell}} Q_{i}(s) Q_{i}^{-1}(0)-1\right) / s\right\|_{\infty} \\
& =\left\|\left(\frac{P_{i}(s)}{(1+\varepsilon s)^{\ell}} Q_{i, \mathrm{opt}}(s) Q_{i, \mathrm{opt}}^{-1}(0)-1\right) / s\right\|_{\infty}
\end{aligned}
$$

and the corresponding optimal $Q_{i \text {,opt }} \in \mathcal{H}_{\infty}$ solving this problem. Note that optimal solution is not unique: if $Q_{i, o}$ is a solution of 13 , then so is $K Q_{i, o}$, for any non-zero constant $K$. Therefore, we define the normalized free parameter $\widetilde{Q}_{i}(s)=Q_{i}(s) Q_{i}^{-1}(0)$, and try to find optimal $\widetilde{Q}_{i}$ in the problem (14) defined below. First let $\mathcal{H}_{\infty}^{o}=\left\{\widetilde{Q}_{i} \in \mathcal{H}_{\infty}: \widetilde{Q}_{i}(0)=1\right\}$. Note that

$$
\begin{aligned}
\psi_{o} & =\inf _{\widetilde{Q}_{i} \in \mathcal{H}_{\infty}^{o}}\left\|\left(\frac{P_{i}(s)}{(1+\varepsilon s)^{\ell}} \widetilde{Q}_{i}(s)-1\right) / s\right\|_{\infty} \\
& \geq \inf _{\widetilde{Q}_{i} \in \mathcal{H}_{\infty}}\left\|\left(\frac{P_{i}(s)}{(1+\varepsilon s)^{\ell}} \widetilde{Q}_{i}(s)-1\right) / s\right\|_{\infty}=: \widetilde{\psi}_{o} .
\end{aligned}
$$

But the optimal solution of the problem defining $\widetilde{\psi}_{o}$ must lie in $\mathcal{H}_{\infty}^{o}$, because $\left(\frac{P_{i}(s)}{(1+\varepsilon s)^{\ell}} \widetilde{Q}_{i}(s)-\right.$ $1) / s$ is in $\mathcal{H}_{\infty}$ only if $\widetilde{Q}_{i}(0)=1$. Therefore, $\widetilde{\psi}_{o}=\psi_{o}$ and $\widetilde{Q}_{i, \mathrm{opt}}$ is the optimal solution of

$$
\psi_{o}=\inf _{\widetilde{Q}_{i} \in \mathcal{H}_{\infty}}\left\|\left(\frac{P_{i}(s)}{(1+\varepsilon s)^{\ell}} \widetilde{Q}_{i}(s)-1\right) / s\right\|_{\infty} .
$$

The problem 14 is a one-block $\mathcal{H}_{\infty}$ optimization problem, which can be seen as equivalent to a weighted sensitivity minimization for a stable plant with the sensitivity weigh being an integrator. For a general inner function $P_{i}$, the problem 14 can be solved using the techniques developed for the $\mathcal{H}_{\infty}$ control of infinite dimensional systems, see e.g. [6, 10, 13] and their references. It turns out that the optimal $\widetilde{Q}_{i}$ is in the form

$$
\widetilde{Q}_{i, \mathrm{opt}}(s)=\frac{(1+\varepsilon s)^{\ell}}{(1+\delta s)^{(\ell+1)}}\left(\frac{1+\psi_{o}^{2} s^{2}}{P_{i}(s)+\psi_{o} s}\right)
$$

where $\delta \rightarrow 0$ and $\psi_{o}$ is the largest value of $\psi>0$ for which we have

$$
P_{i}(j / \psi)=-j .
$$


Choosing $Q_{1}$ as in (12) with $Q_{i}=K_{1} \widetilde{Q}_{i, \text { opt }}$, for an arbitrary $K_{1} \neq 0$, and defining the controller $C_{1}=Q_{1} /\left(1-P Q_{1}\right)$, we obtain

$$
C_{1}(s)=\frac{K_{1} P_{o}^{-1}(s)}{\frac{(1+\delta s)^{\ell+1}\left(\psi_{o} s+P_{i}(s)\right)}{1+\psi_{o}^{2} s^{2}}-K_{1} P_{i}(s)} .
$$

$\rho=k_{i} K_{1}<\psi_{o}^{-1}$. Hence, depending on the gain $K_{1}$ used in $C_{1}$ we get an allowable range for $k_{i}$,

$$
\left|k_{i}\right|<\psi_{o}^{-1} /\left|K_{1}\right| \text {. }
$$

Note that $\psi_{o}$ is invariant and completely determined by the inner part $P_{i}(s)$ of the plant.

Another interesting problem in this context is to investigate PD (proportional plus derivative) type of $\widetilde{Q}_{i}(s)=\left(1+k_{d} s\right)$ in 14. More precisely,

$$
\psi_{p d}:=\inf _{k_{d} \in \mathbb{R}}\left\|\left(\frac{P_{i}(s)}{(1+\varepsilon s)^{\ell}}\left(1+k_{d} s\right)-1\right) / s\right\|_{\infty}=\inf _{k_{d} \in \mathbb{R}}\left\|\frac{f(s)-1}{s}+k_{d} f(s)\right\|_{\infty} .
$$

where $f(s)=\frac{P_{i}(s)}{(1+\varepsilon s)^{\ell}}$. The function $f$ is in $\mathcal{H}_{\infty}$ and $f(0)=1$. This problem has been studied in the context of resilient PD controller design in [14] and a closed form expression is obtained for the optimal $k_{d}$.

Example (Revisited) For the example given in 11, the equation (16) can be written as

$$
e^{-j h / \psi} m(j / \psi)\left(\frac{1-m(-j / \psi) / 2}{1-m(j / \psi) / 2}\right)=-j
$$

where $m(s)=e^{-2 s} \frac{(1-s)}{(1+s)}$. Since $m(s)$ is inner we have $m(j / \psi)=e^{-j \theta_{m}}$, where $\theta_{m}=$ $2 / \psi+2 \tan ^{-1}(1 / \psi)$. We also have $\frac{1-m(-j / \psi) / 2}{1-m(j / \psi) / 2}=e^{-j \theta}$,

where $\theta=2 \tan ^{-1}\left(\frac{\sin \left(\theta_{m}\right)}{2-\cos \left(\theta_{m}\right)}\right)$. Therefore $\psi_{o}^{-1}$ is the smallest $x$ satisfying

$$
\left(1+\frac{h}{2}\right) x+\tan ^{-1}(x)+\tan ^{-1} \Omega(x)=\frac{\pi}{4}
$$

where $\Omega(x)=\frac{\sin \left(2\left(x+\tan ^{-1}(x)\right)\right)}{2-\cos \left(2\left(x+\tan ^{-1}(x)\right)\right)}$.

We should also note that if we change the inner part of the plant to an input/output delay, $P_{i}(s)=e^{-h s}$ (i.e. consider $m(s)=1$ ), then from (16) we get $\psi_{o}^{-1}=\pi / 2 h$, which is precisely the gain margin of the feedback system whose open loop transfer function is $e^{-h s} / s$.

When we consider a PD type of $\widetilde{Q}_{i}(s)=\left(1+k_{d} s\right)$, the solution of 18 gives optimal $\psi_{p d}^{\mathrm{opt}}$ and the corresponding $k_{d}^{\mathrm{opt}}$. Figure 2 illustrates how the optimal $\psi_{o}, \psi_{p d}^{\mathrm{opt}}$ and $k_{d}^{\mathrm{opt}}$ vary with $h$. Note from this figure that the use of PD term does not lead to significant performance degradation (reduction in the largest allowable $k_{i}$ range) compared to the use of optimal $\widetilde{Q}_{i}$ of 15 .

\subsection{Extension to Unstable Plants}

Now consider unstable SISO plants factorized as

$$
P(s)=\frac{N(s)}{D_{i}(s)}, \quad N(s)=N_{i}(s) N_{o}(s)
$$




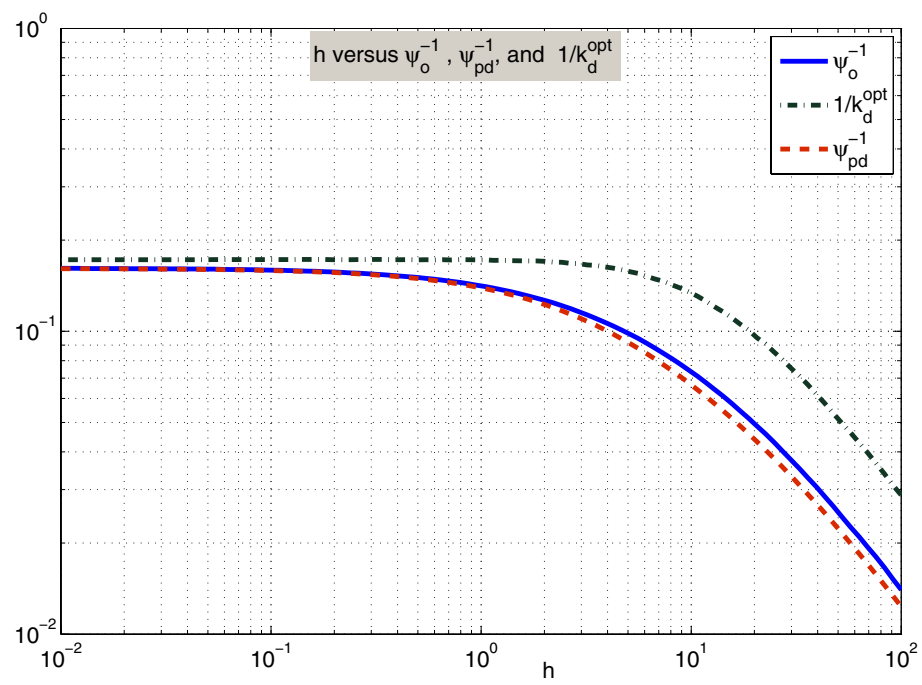

Fig. 2. $h$ versus $\psi_{o}^{-1}, \psi_{p d}^{-1}$ and $1 / k_{d}^{\text {opt }}$.

where $N_{o}(s)$ is outer, $N_{i}(s)$ and $D_{i}(s)$ are inner with $D_{i}$ being a finite Blaschke product (i.e. the plant has finitely many poles in $\mathbb{C}_{+}$, and it has no poles on the Im-axis). As before, we will assume that $N_{i}(0)=1$. For this type of plants $C_{1} \in \mathcal{S}(P)$ if and only if

$$
C_{1}(s)=\frac{X(s)+D_{i}(s) Q_{1}(s)}{Y(s)-N(s) Q_{1}(s)} \text { for some } Q_{1} \in \mathcal{H}_{\infty}
$$

where $X, Y \in \mathcal{H}_{\infty}$ satisfy

$$
Y(s)=\frac{1-N(s) X(s)}{D_{i}(s)} .
$$

Let $p_{1}, \ldots, p_{n}$ be the zeros of $D_{i}(s)$, i.e. poles of $P(s)$ in $\mathbb{C}_{+}$, and for simplicity of the exposition assume that they are distinct. Then, $Y \in \mathcal{H}_{\infty}$ if and only if the function $X \in \mathcal{H}_{\infty}$ satisfy $X\left(p_{i}\right)=1 / N\left(p_{i}\right), i=1, \ldots, n$. If we use $C_{1}$ in the form of 21] as the initial stabilizing controller for the plant $P$, then

$$
T_{1}(s)=N(s)\left(X(s)+D_{i}(s) Q_{1}(s)\right) .
$$

Therefore $\psi=\left\|\left(T_{1}(s) T_{1}^{-1}(0)-1\right) / s\right\|_{\infty}$ is obtained as

$$
\psi=\left\|\frac{N(s) N(0)^{-1} Q_{1 X}(s) Q_{1 X}(0)^{-1}-1}{s}\right\|_{\infty},
$$

where $Q_{1 X}(s)=\left(X(s)+D_{i}(s) Q_{1}(s)\right)$. Thus the optimal $\psi_{o}$ is the smallest $\psi$ over $Q_{1 X}(s)=\left(X(s)+D_{i}(s) Q_{1}(s)\right)$ for $Q_{1} \in \mathcal{H}_{\infty}$. Define

$$
Q_{1 X}(s)=: \frac{N_{o}^{-1}(s)}{(1+\varepsilon s)^{\ell}} Q_{X}(s) \text { where } \varepsilon \searrow 0,
$$


and $\ell$ is the relative degree of $N_{o}(s)$. Then, we have an invertible relation between the free parameters $Q_{1 X}$ and $Q_{X}$ in $\mathcal{H}_{\infty}$. Note that the problem 23] is exactly in the form 13] except that $Q_{1 X}(s)$ is restricted to have $Q_{1 X}\left(p_{i}\right)=X\left(p_{i}\right)=1 / N\left(p_{i}\right)$, whereas in (13) there is no such restriction on the free parameter $Q_{i} \in \mathcal{H}_{\infty}$. In summary, we have the following

$$
\begin{gathered}
\psi\left(Q_{X}\right):=\left\|s^{-1}\left(\frac{N_{i}(s)}{(1+\varepsilon s)^{\ell}} \frac{Q_{X}(s)}{Q_{X}(0)}-1\right)\right\|_{\infty} \\
\psi_{o}=\inf \left\{\psi\left(Q_{X}\right): Q_{X} \in \mathcal{H}_{\infty} \text { and } Q_{X}\left(p_{i}\right)=\frac{\left(1+\varepsilon p_{i}\right)^{\ell}}{N_{i}\left(p_{i}\right)}, i=1 \ldots, n\right\} .
\end{gathered}
$$

As in Section 3.1 we will be restricting ourselves to $Q_{X} \in \mathcal{H}_{\infty}$ such that $Q_{X}(0)=1$, because $\psi\left(K Q_{X}\right)=\psi\left(Q_{X}\right)$ for any non-zero $K$. Thus, in the unstable plants case the problem is modified to finding

$$
\begin{aligned}
\psi_{o}= & \inf _{\widetilde{Q}_{X}}\left\|s^{-1}\left(\frac{N_{i}(s)}{(1+\varepsilon s)^{\ell}} \widetilde{Q}_{X}(s)-1\right)\right\|_{\infty} \\
& \text { subject to } \widetilde{Q}_{X} \in \mathcal{H}_{\infty} \text { and } \widetilde{Q}_{X}\left(p_{i}\right)=\frac{\left(1+\varepsilon p_{i}\right)^{\ell}}{N_{i}\left(p_{i}\right)}, i=1 \ldots, n .
\end{aligned}
$$

For a given $\gamma>\psi_{o}$, the set of all $\widetilde{Q}_{X} \in \mathcal{H}_{\infty}$ satisfying

$$
\left\|s^{-1}\left(\frac{N_{i}(s)}{(1+\varepsilon s)^{\ell}} \widetilde{Q}_{X}(s)-1\right)\right\|_{\infty} \leq \gamma
$$

can be characterized as

$$
\mathcal{Q}_{\gamma}=\left\{\widetilde{Q}_{X}(s)=\frac{F_{1}(s)+F_{2}(s) U(s)}{F_{3}(s)+F_{4}(s) U(s)} \quad: U \in \mathcal{H}_{\infty},\|U\|_{\infty} \leq 1\right\}
$$

where $F_{1}, \ldots, F_{4}$ are computed explicitly from the problem data, see e.g. [6]. Therefore, the problem at hand can be transformed to finding the smallest $\gamma$ for which there exists $U \in \mathcal{H}_{\infty}$, $\|U\|_{\infty} \leq 1$ such that

$$
\frac{F_{1}\left(p_{i}\right)+F_{2}\left(p_{i}\right) U\left(p_{i}\right)}{F_{3}\left(p_{i}\right)+F_{4}\left(p_{i}\right) U\left(p_{i}\right)}=\frac{\left(1+\varepsilon p_{i}\right)^{\ell}}{N_{i}\left(p_{i}\right)}=: \alpha_{i}
$$

for $i=1 \ldots, n$. This leads to a set of interpolation conditions on $U$

$$
U\left(p_{i}\right)=\frac{\alpha_{i} F_{3}\left(p_{i}\right)-F_{1}\left(p_{i}\right)}{F_{2}\left(p_{i}\right)-\alpha_{i} F_{4}\left(p_{i}\right)}=: \beta_{i}
$$

for $i=1 \ldots, n$. For each fixed $\gamma$ we can find $\beta_{i}$ using for example [6]. Now we need to check whether there exists $U \in \mathcal{H}_{\infty}$ with $\|U\|_{\infty} \leq 1$ such that $U\left(p_{i}\right)=\beta_{i}$. This is a Nevanlinna-Pick interpolation problem and it can be solved from the given problem data $\left\{\left(p_{1} \ldots, p_{n}\right),\left(\beta_{1}, \ldots, \beta_{n}\right)\right\}$, see e.g. [6, 11, 18].

In summary, for unstable plants the problem is solved in two steps:

1. Given $\gamma>\psi_{o}$, solve the suboptimal version (26) of the problem 13 studied in Section 3.1 characterize all suboptimal solutions in the form 27), i.e. find $F_{1}, F_{2}, F_{3}, F_{4}$.

2. Given $p_{1}, \ldots, p_{n}$, determine $\beta_{1}, \ldots, \beta_{n}$ from the first step. Use this data to check if the Nevanlinna-Pick interpolation problem has a feasible solution. If yes decrease $\gamma$, if no increase $\gamma$, and repeat Steps 1 and 2; using a bisection in this iteration find the optimal $\gamma_{o}$. For $\gamma=\gamma_{o}+\epsilon$, where $\epsilon>0$, the Nevanlinna-Pick problem gives a solution $U$, which in turn gives our suboptimal $Q_{X}$, from which we get $Q_{1 X}$ and hence $C_{1}$. 
Example. Let $h>0$, and consider $P(s)=\frac{e^{-h s}}{s+2}\left(\frac{s+1+2(s-1) e^{-2 s}}{s+3-5 e^{-0.5 s}}\right)$. This plant is unstable with single pole $p_{1}=0.6367$ in $\mathbb{C}_{+}$. Therefore, its factorization can be done as 20 where

$$
\begin{aligned}
D_{i}(s) & =\frac{s-0.6367}{s+0.6367} \\
N_{i}(s) & =e^{-h s} \frac{(s+1)+2(s-1) e^{-2 s}}{(1-s) e^{-2 s}-2(s+1)} \\
N_{o}(s) & =\frac{(1-s) e^{-2 s}-2(s+1)}{(s+2)\left(s+3-5 e^{-0.5 s}\right)} D_{i}(s) .
\end{aligned}
$$

For $h=3$ we have $\psi_{o}=8.6744$. This gives $\alpha_{1}=N_{i}\left(p_{1}\right)^{-1}=-14.945$. Applying the procedure described above we find $F_{1}, \ldots, F_{4}$ for each fixed $\gamma>\psi_{o}$, and compute $\beta_{1}$ defined by 29]. Since we have single interpolation condition, the solution of Nevanlinna-Pick problem is rather trivial: it is solvable if and only if $\left|\beta_{1}\right| \leq 1$, and as a solution we can take $U(s)=\beta_{1}$. By using a bi-section search we find that smallest $\gamma>\psi_{o}$ leading to $\left|\beta_{1}\right| \leq 1$ is $\gamma_{o}=13.4485$, which leads to $\beta_{1}=-1$, see Figure 3 Thus if we choose $U(s)=-1$, we get

$$
\widetilde{Q}_{X}(s)=\frac{F_{1}(s)-F_{2}(s)}{F_{3}(s)-F_{4}(s)},
$$

where $F_{1}, \ldots, F_{4}$ are computed from the solution of the suboptimal one-block $\mathcal{H}_{\infty}$ problem with $\gamma=13.45>\gamma_{o}$.

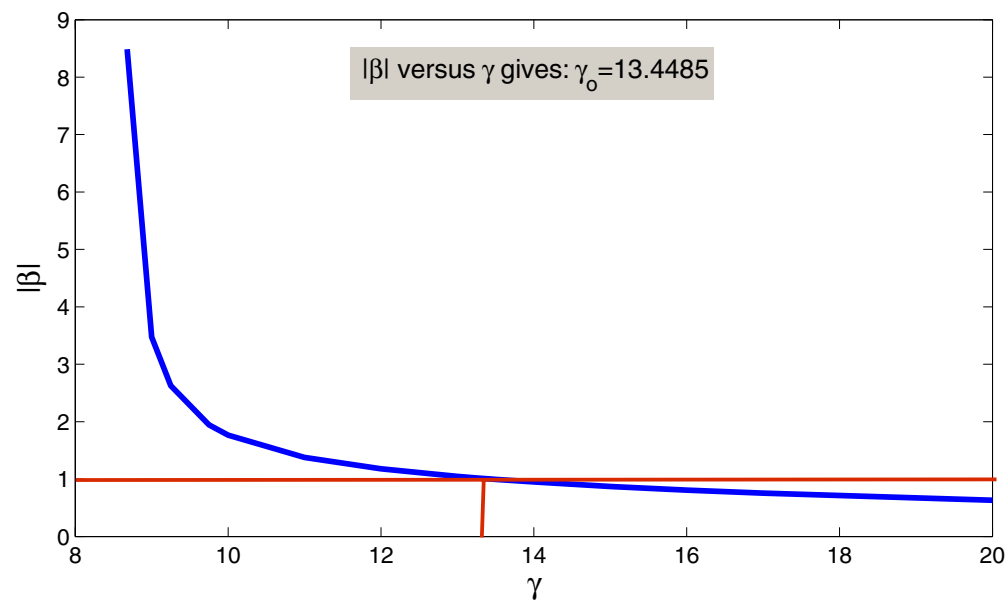

Fig. 3. $|\beta|$ versus $\gamma$. 


\section{Conclusions}

A sufficient condition is derived for $C=C_{1} C_{p i}$, cascade connection of a PI controller $C_{p i}$, and an initial stabilizing controller $C_{1}$, to stabilize a given plant $P$. Design of $C_{1}$ for the largest allowable range of the integral action gain interval is investigated for stable plants, including systems with internal and input-output delays. We used parametrization of all stabilizing controller to characterize $C_{1}$. Then we have seen that the problem at hand reduces to a weighted sensitivity minimization for a stable plant whose inner part is infinite dimensional and the weight is an integrator. When we consider a PD-like $\tilde{Q}_{i}(s)$ in the parametrization of $C_{1}$, the problem becomes finding optimal $k_{d}$ in [18], which is solved in [14].

For unstable plants the problem of finding the largest allowable range of the integral action gain is solved in two steps. First the a suboptimal one-block problem is solved and in the second step a Nevanlinna-Pick interpolation problem is solved.

We should also point out that the result stated in Section 2 is a sufficient condition. Therefore the largest allowable integral action gain found in Section 3 is within the set of allowable gains characterized by this sufficient condition, which may be conservative. It would be interesting to investigate the level of conservatism in this approach. We leave this open problem to a future study.

\section{APPENDIX}

Proof of Theorem 11 a) Let $P=\tilde{Y}^{-1} \tilde{X}$ be a left-coprime-factorization (LCF) of $P$ and let $N_{1} D_{1}^{-1}$ be a right-coprime-factorization (RCF) of $C_{1}$. Since $C_{1}$ stabilizes $P, M_{1}:=$ $\tilde{Y} D_{1}+\tilde{X} N_{1}$ is unimodular in $\mathcal{H}_{\infty}^{r \times r}$. With $C_{1} \in \mathcal{S}(P)$, we have $Q_{1}:=C_{1}\left(I+P C_{1}\right)^{-1} \in$ $\mathcal{H}_{\infty}^{r \times r}$ and $T_{1}:=P C_{1}\left(I+P C_{1}\right)^{-1}=P Q_{1} \in \mathcal{H}_{\infty}^{r \times r}$. Now $C_{y}=I-C_{x}$ stabilizes $T_{1}$ if and only if $C_{y}\left(I+T_{1} C_{y}\right)^{-1} \in \mathcal{H}_{\infty}^{r \times r}$, which implies $\left(I+T_{1} C_{y}\right)^{-1} \in \mathcal{H}_{\infty}^{r \times r}$. Define $D_{c}:=\left(I+T C_{y}\right)^{-1} D_{1}, N_{c}=N_{1}+Q_{1} C_{y} D_{c}$; then $N_{c}, D_{c} \in \mathcal{H}_{\infty}^{r \times r}$. Write $C=C_{1} C_{x}$ as $C=C_{1}+C_{1} C_{y}=N_{c} D_{c}^{-1}$. Then $\tilde{Y} D_{c}+\tilde{X} N_{c}=\tilde{Y} D_{c}+\tilde{X}\left[N_{1}+Q_{1} C_{y} D_{c}\right]=\tilde{Y} D_{c}+$ $\tilde{X} N_{1}+\tilde{Y} P Q_{1} C_{y} D_{c}=\tilde{Y}\left(I+T_{1} C_{y}\right) D_{c}+\tilde{X} N_{1}=\tilde{Y}\left(I+T_{1} C_{y}\right)\left(I+T C_{y}\right)^{-1} D_{1}+\tilde{X} N_{1}=$ $M_{1}$ is unimodular and hence, $C=C_{1} C_{x} \in \mathcal{S}(P)$.

b) Let $P=X Y^{-1}$ be an RCF and $C_{1}=\tilde{D}_{1}^{-1} \tilde{N}_{1}$ be an LCF. Then $C_{1} \in \mathcal{S}(P)$ if and only if $\tilde{M}_{1}:=\tilde{D}_{1} Y+\tilde{N}_{1} X$ is unimodular; hence, $\operatorname{det} \tilde{M}_{1}(0) \neq 0$. Since $P, C_{1} \in \mathcal{S}(P)$ do not have transmission-zeros at $s=0, \operatorname{det} X(0) \neq 0$ and $\operatorname{det} \tilde{N}_{1}(0) \neq 0$. Since $\operatorname{det} T_{1}(0)=$ $\operatorname{det} X(0) \tilde{M}_{1}(0) \tilde{N}_{1}(0) \neq 0$, we conclude that $T_{1} \in \mathcal{H}_{\infty}^{r \times r}$ does not have transmission-zeros at $s=0$. It follows from [9], Proposition 2, that the PID-controller $\hat{C}_{\text {pid }}$ in (4) stabilizes $T_{1} \in \mathcal{H}_{\infty}^{r \times r}$. Therefore, by (1), $C_{1} C_{x} \in \mathcal{S}(P)$, where $C_{x}=I+\hat{C}_{p i d}$.

\section{References}

1. Bélanger, P.R.: Control engineering: A modern approach. Saunders College Pub., Philadelphia (1995)

2. Davison, E.J.: The robust control of a servomechanism problem for linear time-invariant multivariable systems. IEEE Transactions on Automatic Control 21, 25-34 (1976)

3. Davison, E.J., Özgüner, Ü.: Expanding system problem. Systems and Control Letters 1, 255-260 (1982)

4. Desoer, C.A., Wang, Y.T.: On the minimum order of a robust servocompensator. IEEE Transactions on Automatic Control 23, 70-73 (1978) 
5. Engelborghs, K., Luzyanina, T., Roose, D.: Numerical bifurcation analysis of delay differential equations using DDE-BIFTOOL. ACM Transactions on Mathematical Software 28, 1-21 (2002)

6. Foias, C., Özbay, H., Tannenbaum, A.: Robust control of infinite dimensional systems: Frequency domain methods. LNCIS, vol. 209. Springer, London (1996)

7. Francis, B.A., Wonham, W.M.: The internal model principle of control theory. Automatica 12, 457-465 (1976)

8. Gümüşsoy, S., Özbay, H.: Remarks on $H^{\infty}$ controller design for SISO plants with time delays. In: Proc. 5th IFAC Symposium on Robust Control Design, Toulouse, France (2006)

9. Gündeş, A.N., Özbay, H., Özgüler, A.B.: PID controller synthesis for a class of unstable MIMO plants with I/O delays. Automatica 43, 135-142 (2007)

10. Kashima, K., Özbay, H., Yamamoto, Y.: A Hamiltonian-based solution to the mixed sensitivity optimization problem for stable pseudorational plants. Systems \& Control Letters 54, 1063-1068 (2005)

11. Krein, M.G., Nudel'man, A.A.: The Markov moment problem and extremal problems. Translations of Mathematical Monographs 50 (1977)

12. Olgaç, N., Sipahi, R.: A practical method for analyzing the stability of neutral type LTItime delayed systems. Automatica 40, 847-853 (2004)

13. Özbay, H.: A simpler formula for the singular values of a certain Hankel operator. Systems \& Control Letters 15, 381-390 (1990)

14. Özbay, H., Gündeş, A.N.: Resilient PI and PD controller designs for a class of unstable plants with I/O delays. Applied and Computational Mathematics 6, 18-26 (2007)

15. Toker, O., Özbay, H.: $H^{\infty}$ optimal and suboptimal controllers for infinite dimensional SISO plants. IEEE Transactions on Automatic Control 40, 751-755 (1995)

16. Vidyasagar, M.: Control system synthesis: A factorization approach. MIT Press, Cambridge (1985)

17. Vyhlidal, T., Zitek, P.: Mapping the spectrum of a retarded time-delay system utilizing root distribution features. In: Proc. IFAC Workshop on Time-Delay Systems, L'Aquila, Italy (2006)

18. Zeren, M., Özbay, H.: Comments on Solutions to combined sensitivity and complementary sensitivity problem in control systems. IEEE Transactions on Automatic Control 43, 724 (1998) 